This item was submitted to Loughborough's Research Repository by the author.

Items in Figshare are protected by copyright, with all rights reserved, unless otherwise indicated.

\title{
The acute effect of dopamine infusion on lipid and cytokine concentrations in persons with a cervical spinal cord injury - a pilot study
}

\section{PLEASE CITE THE PUBLISHED VERSION}

https://doi.org/10.1038/s41393-021-00613-9

\section{PUBLISHER}

Springer Nature [academic journals on nature.com]

\section{VERSION}

AM (Accepted Manuscript)

\section{PUBLISHER STATEMENT}

This paper was accepted for publication in the journal Spinal Cord and the definitive published version is available at https://doi.org/10.1038/s41393-021-00613-9.

\section{LICENCE}

CC BY-NC-ND 4.0

\section{REPOSITORY RECORD}

Hoekstra, Sven, Yoshi-ichiro Kamijo, Takayoshi Matsushita, Christof Leicht, Kazunari Nishiyama, Vicky Goosey-Tolfrey, Yasunori Nagono, et al.. 2021. "The Acute Effect of Dopamine Infusion on Lipid and Cytokine Concentrations in Persons with a Cervical Spinal Cord Injury - a Pilot Study". Loughborough University. https://hdl.handle.net/2134/13514152.v1. 


\title{
The acute effect of dopamine infusion on lipid and cytokine concentrations in persons with a cervical spinal cord injury - a pilot study
}

\author{
Sven P. Hoekstra ${ }^{1,2}$, Yoshi-ichiro Kamijo ${ }^{1}$, Takayoshi Matsushita ${ }^{1}$, Christof A. Leicht ${ }^{2}$, Kazunari \\ Nishiyama $^{1}$, Victoria L. Goosey-Tolfrey ${ }^{2}$, Yasunori Nagano ${ }^{3}$, Yasunori Umemoto ${ }^{1}$, Masumi \\ Nakahama $^{1}$, Yohei Furotani ${ }^{1}$, Fumihiro Tajima ${ }^{1,2}$ \\ 1. Department of Rehabilitation Medicine; Wakayama Medical University; Wakayama; Japan \\ 2. The Peter Harrison Centre for Disability Sport; School of Sport, Exercise, and Health Sciences; \\ Loughborough University; Loughborough; United Kingdom \\ 3. Department of Rehabilitation Center; Kochi Medical School Hospital; Nankoku; Japan
}

Running title: Dopamine infusion in persons with CSCI

\section{Corresponding author}

Dr. Yoshi-Ichiro Kamijo M.D., Ph.D.

Department of Rehabilitation Medicine; Wakayama Medical University

811-1 Kimiidera Wakayama City; Wakayama 641-8509, Japan

+81-73-441-0664

yoshikmj@.gmail.com 
Study design Acute experimental study

Objectives To investigate the acute response of markers of lipid metabolism and interleukin (IL)-6 to dopamine infusion in people with a cervical spinal cord in injury (CSCI).

Setting Laboratory of Wakayama Medical University, Japan

Methods Ten participants, four with CSCI and six AB individuals, underwent 50-min of dopamine infusion. Blood samples were collected prior to, immediately after and $1 \mathrm{~h}$ following cessation of dopamine infusion for the determination of circulating catecholamine, lipid, ketone body and IL-6 concentrations.

Results The adrenaline concentration following dopamine infusion was increased by $59 \pm 7 \%$ in CSCI ( $p=0.038$, Cohen`s d effect size (ES): 1.47), while this was not changed in $\mathrm{AB}(p=0.223)$. Triglycerides and acetoacetic acid concentration were increased in both groups, immediately after and concentration in either group ( $p \geq 0.368)$.

Conclusions Dopamine infusion induced a sustained increase in triglyceride and ketone body concentrations in persons with CSCI. In contrast, cytokine concentrations were not affected by dopamine infusion. These findings suggest that circulating catecholamines can stimulate metabolism in people with CSCI despite the presence of autonomic dysfunction. 
Persons with a cervical spinal cord injury (CSCI) have a lower fasting high-density

lipoprotein and higher triglyceride concentration compared with able-bodied (AB) individuals [1], suggesting sub-optimal lipid metabolism. This may be related to lifestyle factors as well as secondary complications resulting from the injury, such as sympathetic dysfunction [2]. Nonetheless, the significance of sympathetic activation and circulating catecholamines for lipid metabolism in persons with CSCI is unclear. For instance, circulating catecholamines are widely believed to play a crucial role in catabolic processes such as lipolysis [3]. Furthermore, blocking sympathetic nerve activation attenuates lipolysis and leads to an increased fat mass in animals [4]. Notwithstanding this evidence pointing at the importance of sympathetic activation for lipid metabolism, however, persons with CSCI show a similar postprandial lipolytic rate compared with able-bodied (AB) individuals [5] despite chronically low concentrations of circulating catecholamines and decentralised adipose tissue below the spinal lesion [6]. This indicates that some aspects of lipid metabolism may not be affected by the autonomic dysfunction found following CSCI. Dopamine, a member of the catecholamine family, acts on $\alpha$ - and $\beta$-adrenergic as well as dopaminergic receptors [7]. In addition, dopamine is a precursor of adrenaline and noradrenaline [8]. Similar to adrenaline and noradrenaline, infusion of dopamine in resting AB individuals elevates circulating concentrations of triglycerides, free fatty acids and glycerol [9], suggesting an increase in lipolysis [3]. In persons with CSCI, autonomic dysfunction may lead to peripheral adaptations that alter the response to circulating catecholamines. For instance, an exacerbated blood pressure response has been reported after noradrenaline infusion in this population, suggested to be the result of $\alpha$ adrenergic receptor hypersensitivity [10]. However, the metabolic response to catecholamine administration in persons with loss of supraspinal autonomic control of adipose tissue below the spinal lesion and chronically low circulating catecholamine concentrations has not yet been studied. Similarly, while dopamine infusion can increase ketone body concentration in AB persons [11], this response has yet to be investigated in individuals with SCI. The circulating concentration of ketone bodies (comprising of acetoacetic acid, 3-hydroxybutyric acid and acetone) reflect the balance 
between ketogenesis by the liver and uptake by peripheral tissue, with skeletal muscle as a primary site for ketone body clearance [12]. As such, in addition to the neurological dysfunction in persons with CSCI, their smaller muscle mass may affect ketone metabolism by a reduced oxidation of ketone bodies by skeletal muscle following dopamine infusion. This may further impact on persons with CSCI: while a modest elevation in circulating ketone bodies can form a substrate for lipogenesis or induce positive effects on oxidative stress and neuronal health, an exacerbated rise in ketone body concentration as a result of impaired clearance capacity may ultimately lead to ketoacidosis [12].

Apart from their effect on lipid metabolism, catecholamines are also suggested to stimulate cytokine production $[13,14]$. For instance, in-vitro, dopamine increases interleukin-6 (IL-6) production in epidermal cells [13], while adrenaline infusion in resting AB individuals at similar concentrations as observed during exercise elevates plasma IL-6 concentration [14]. Interleukin-6 is a pleiotropic cytokine; elevated resting concentrations are associated with chronic diseases such as type II diabetes mellitus, but transient, short-term elevations may have beneficial effects by stimulating the production of anti-inflammatory cytokines such as interleukin-1 receptor antagonist (IL-1ra) [15]. Indeed, the acute elevation of IL-6 concentration after acute exercise has been suggested key for the anti-inflammatory effects of regular exercise training [16]. Supporting the importance of sympathetic activation for IL-6 production, the absence of an acute elevation in catecholamine concentrations after exercise in persons with CSCI is accompanied by an attenuated IL-6 response compared with individuals with paraplegia or non-SCI impairments [17]. In keeping with markers of lipid metabolism, however, it is currently unknown whether catecholamine administration can independently increase circulating cytokine concentration in persons with CSCI.

The aims of this pilot study were twofold: to 1) investigate the acute effects of dopamine infusion on circulating concentrations of triglycerides and ketone bodies, and 2) to investigate the acute cytokine response to dopamine infusion. It was hypothesised that, despite autonomic dysfunction, dopamine infusion leads to increased plasma triglyceride, ketone body as well as IL-6 and IL-1ra concentration in persons with CSCI. As an additional aim, the safety of dopamine administration was assessed by monitoring hemodynamic measures. Considering the lack of previous 
investigations and the potential harmful effects of catecholamine administration in persons with CSCI, dopamine infusion was chosen as it has a more modest effect on the cardiovascular system compared with adrenaline and noradrenaline [9].

\section{Methods}

Twelve adult males volunteered to participate in this study; six with chronic CSCI (age: $39 \pm 10$ yrs, height: $\quad 175 \pm 4 \mathrm{~cm}$, body mass: $62.3 \pm 13.4 \mathrm{~kg}$, American Spinal Injury Association Impairment Scale: $\mathrm{A}(\mathrm{n}=3)$ and $\mathrm{B}(\mathrm{n}=1)$, and six age-matched $\mathrm{AB}$ males (age: $36 \pm 5 \mathrm{yrs}$, height: $171 \pm 9 \mathrm{~cm}$, body mass: $66.8 \pm 9.7 \mathrm{~kg}$ ). All procedures of this study were approved by the local ethics committee of Wakayama Medical University (project number: 1648), for which all participants signed informed consent prior to participation.

\section{Procedures}

Participants arrived in the laboratory after a $5 \mathrm{~h}$ fast, having abstained from caffeine and alcohol for at least $12 \mathrm{~h}$. Following voiding of the bladder, participants rested for 10 min before the first blood sample was collected from a cannula inserted into an antecubital vein of the arm.

Thereafter, dopamine hydrochloride dissolved in saline was infused into a saphenous vein in the foot at a rate of $1.25 \mu \mathrm{g} / \mathrm{kg} / \mathrm{min}$ for $20 \mathrm{~min}$, followed by $20 \mathrm{~min}$ at $2.5 \mu \mathrm{g} / \mathrm{kg} / \mathrm{min}$ and $10 \mathrm{~min}$ at 5.0 $\mu \mathrm{g} / \mathrm{kg} / \mathrm{min}$. Blood pressure was measured every min at the brachial artery (STBP-780, Colin, Komaki, Japan), while heart rate was measured continuously using a 3-lead electrocardiogram (PhysioFlow Lab-1, Manatec Biomedical, Paris, France). Blood pressure and heart rate data were averaged over the final five min of baseline recordings and each infusion stage. Immediately following cessation of dopamine infusion, a second blood sample was drawn, and participants rested for another $60 \mathrm{~min}$. Finally, a $1 \mathrm{~h}$ post-blood sample was drawn. Participants remained in supine position and did not consume any food or liquid throughout the duration of the experiment. 
Blood samples were divided into EDTA-containing tubes for the determination of adrenaline, noradrenaline, dopamine and glucagon concentrations in plasma and a sodium fluoride-containing tube for glucose concentration, while the remaining blood was allowed to clot for at least $30 \mathrm{~min}$ in serum tubes for the determination of insulin, triglycerides, total cholesterol and the ketone bodies 3hydroxybutyric acid and acetoacetic acid concentrations in serum. Samples were centrifuged for 15 min at $3500 \mathrm{rpm}$ and stored at $-80^{\circ} \mathrm{C}$ until batch analysis. Haematocrit and haemoglobin concentration were measured in triplicate from whole blood, using a cell counter (MEK-6400, Nihon Kohden, Tokyo). All lipids, ketone bodies, glucose, insulin and C-reactive protein (CRP) were analysed by a specialised company (SRL, Tokyo, Japan), while IL-6, IL-1ra and tumour necrosis factor- $\alpha$ (TNF- $\alpha$ ) were analysed in-house using commercially available enzyme-linked immunoassays (R\&D Systems, Abingdon, UK). Plasma and serum markers were corrected for changes in plasma volume according to the method postulated by Dill and Costill [18].

\section{Statistical analyses} tested using the Friedman test, followed by the Wilcoxon signed-rank test for pairwise comparisons between time points when significance was observed. The Mann-Whitney test was used to test for differences between both groups at each time point. Cohen`s d effect sizes (ES) of within-group differences of metabolic markers were calculated using G*Power (HHU, Düsseldorf, Germany). Considering the small sample size included and the exploratory nature of the present study, no Bonferroni-corrections were applied [19]. All analyses were performed using the $23^{\text {rd }}$ version of SPSS and statistical significance was defined as $p<0.05$.

\section{Results}

Inspection of the individual data indicated that the resting glucose $(117 \mathrm{mg} / \mathrm{dl}$ and $128 \mathrm{mg} / \mathrm{dl})$ and insulin concentration $(29.7 \mu \mathrm{UI} / \mathrm{ml}$ and $18.0 \mu \mathrm{UI} / \mathrm{ml})$ of two participants with CSCI were considerably higher when compared with the other group members (i.e. resting glucose and insulin concentration more than 9 SDs and 8 SDs higher than the mean of the other participants with CSCI, 
168 fasting prior to the laboratory visit, both participants were excluded from further analyses.

170 in CSCI compared with $\mathrm{AB}$, while there was no difference in dopamine concentration $(p=0.71)$.

171 There was no difference in glucose and insulin concentration, nor was there a difference in the

172 concentration of total cholesterol, triglycerides, acetoacetic acid or 3-hydroxybutyric acid prior to

173 infusion $(p \geq 0.091)$. There were also no differences between CSCI and AB in any of the cytokines or

174 CRP at rest $(p \geq 0.171)$ (Fig. 1 and 2, Table 1).

175 Table 1. The acute changes in cytokine concentrations and markers of glucose and lipid metabolism following 176 dopamine infusion in CSCI and AB

\begin{tabular}{|c|c|c|c|c|c|c|}
\hline \multirow[b]{2}{*}{ Parameter } & \multicolumn{3}{|c|}{ CSCI } & \multicolumn{3}{|c|}{$\mathrm{AB}$} \\
\hline & Pre & Post & Post $+1 \mathrm{~h}$ & Pre & Post & Post $+1 \mathrm{~h}$ \\
\hline Glucose (mg/dl)* & $94.0 \pm 2.5$ & $102.5 \pm 5.3$ & $90.5 \pm 2.7$ & $94.3 \pm 5.0$ & $103.7 \pm 6.0$ & $96.7 \pm 5.5$ \\
\hline Insulin (mg/dl)* & $3.44 \pm 2.28$ & $8.09 \pm 9.35$ & $2.51 \pm 1.72$ & $2.89 \pm 2.12$ & $5.43 \pm 3.15$ & $2.71 \pm 2.10$ \\
\hline Glucagon (pg/ml) & $149 \pm 20$ & $191 \pm 40$ & $144 \pm 22$ & $136 \pm 30$ & $165 \pm 34$ & $133 \pm 19$ \\
\hline Total cholesterol (mg/dl)* & $150 \pm 22$ & $163.5 \pm 24$ & $152 \pm 23$ & $174 \pm 39$ & $181 \pm 44$ & $175 \pm 40$ \\
\hline IL-6 (pg/ml) & $3.50 \pm 3.92$ & $4.28 \pm 3.59$ & $6.28 \pm 4.39$ & $4.55 \pm 5.47$ & $4.56 \pm 5.20$ & $4.87 \pm 5.04$ \\
\hline IL-1ra (pg/ml) & $479 \pm 84$ & $475 \pm 94$ & $537 \pm 290$ & $476 \pm 230$ & $419 \pm 68$ & $529 \pm 238$ \\
\hline TNF- $\alpha(\mathrm{pg} / \mathrm{ml})$ & $1.35 \pm 0.33$ & $1.18 \pm 0.29$ & $0.90 \pm 0.43$ & $0.92 \pm 0.37$ & $0.70 \pm 0.39$ & $1.07 \pm 0.30$ \\
\hline $\mathrm{CRP}(\mathrm{ng} / \mathrm{ml})^{\wedge}$ & & & $3951 \pm 411$ & & & \\
\hline & $3883 \pm 4083$ & $4239 \pm 4319$ & 4 & $1282 \pm 2247$ & $1521 \pm 2342$ & $1320 \pm 2160$ \\
\hline
\end{tabular}

\section{7}

178

Dopamine infusion

179

The acute changes in plasma catecholamine concentrations are shown in Fig. 1. Plasma

dopamine concentrations in response to dopamine infusion were elevated in both groups $(p \leq 0.001)$.

While the plasma adrenaline concentration was elevated immediately following dopamine infusion in

$\operatorname{CSCI}(p=0.038$, ES: 1.47), there was no increase in $\mathrm{AB}(p=0.223)$. Noradrenaline concentration

AB: 1.85). At $1 \mathrm{~h}$ post-infusion, noradrenaline concentrations returned to baseline in both groups, 
190

191

192

193

194

195

196

197

198

199

200

201

202

203

204

205

206

207

208

209

210

211

212

213

214
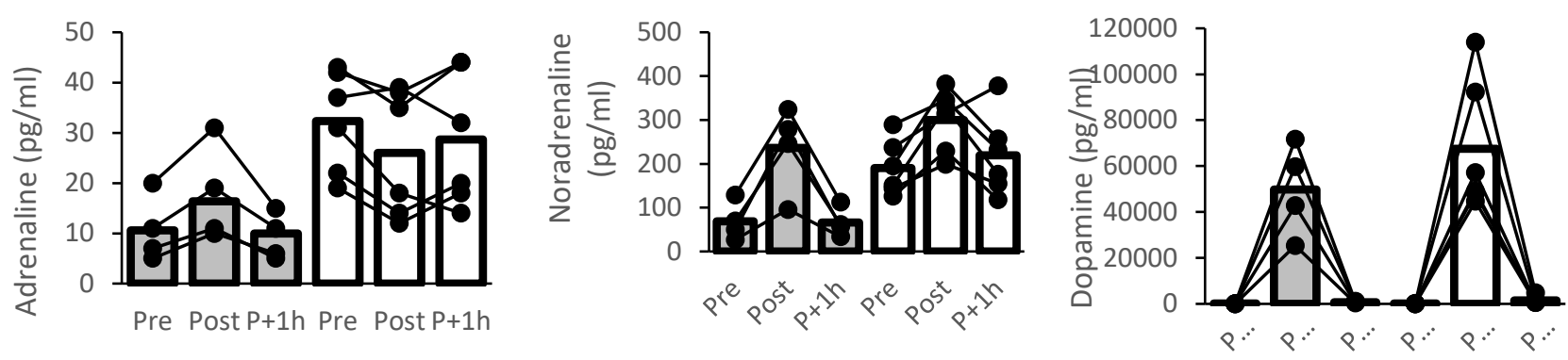

Concentrations of acetoacetic acid were elevated in both groups directly post-infusion, as well as at $1 \mathrm{~h}$ post ( $p \leq 0.030$, ES CSCI: 1.72 , ES AB: 1.31$)$, with no differences between $\mathrm{AB}$ and CSCI ( $p$ $\geq 0.394$ ). 3-Hydroxybutyric acid, however, was elevated immediately post-infusion in CSCI only ( $p=$ 0.039, ES: 1.44; $\mathrm{AB} p=0.115$ ) and remained elevated at $1 \mathrm{~h}$ post (pre versus post $+1 \mathrm{~h} p=0.046$ ) in this group. Total ketone body concentration was elevated in both groups directly post-infusion, as well as at $1 \mathrm{~h}$ post ( $p \leq 0.042$, ES CSCI: 1.36 , ES AB: 0.92), with no differences between AB and CSCI $(p \geq 0.589)$. The concentrations of triglycerides and total cholesterol were increased postinfusion and remained elevated $1 \mathrm{~h}$ thereafter in both groups (triglycerides $p \leq 0.042$, ES CSCI: 1.00, ES AB: 1.12; total cholesterol $p \leq 0.040$, ES CSCI: 1.29, ES AB: 0.40). There were no differences between both groups at any time point (triglycerides $p \geq 0.818$; total cholesterol $p \geq 0.180$ ) (Fig. 2).

Glucose and insulin concentrations were elevated immediately following dopamine infusion in both groups (glucose $p \leq 0.009$, ES CSCI: 2.00, ES AB: 1.61; insulin $p \leq 0.007$, ES CSCI: 0.49, ES AB: 0.93), returning to baseline $1 \mathrm{~h}$ post-infusion. Glucagon, on the other hand, was not elevated in either group (CSCI $p=0.097 ; \mathrm{AB} p=0.069)($ Table 1$)$.
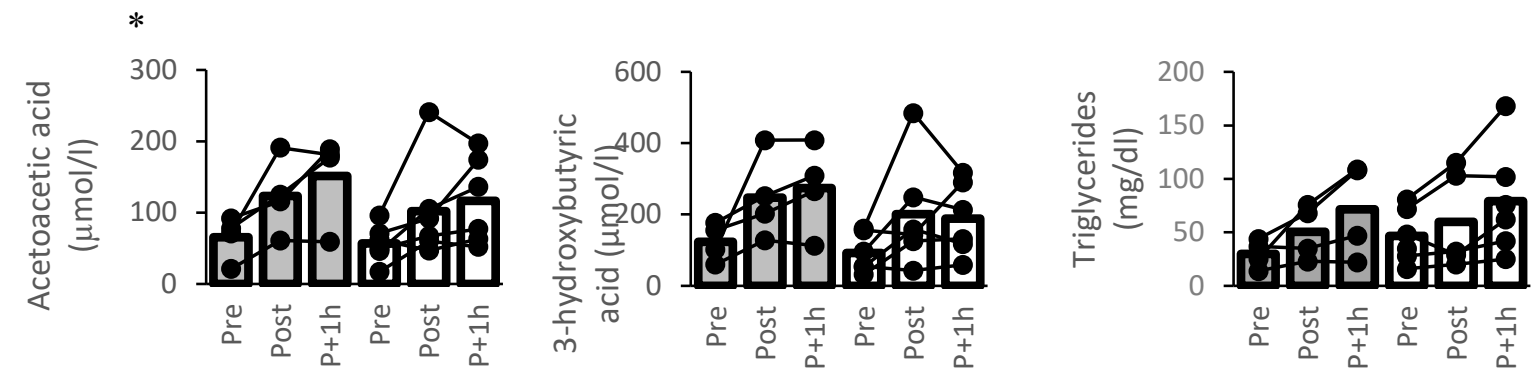

Fig. 2 The acute changes in serum ketone body and triglyceride concentrations following dopamine infusion in CSCI (grey) and AB (white). Lines indicate individual data, while the bars represent group means.

$*$ effect of time $(p<0.05)$. 
infusion did not increase circulating IL-6 or IL-1ra concentrations in either group (IL-6 $p=0.607$; IL-

217 1ra $p \geq 0.069)$. Furthermore, TNF- $\alpha$ concentrations did not change following infusion $(p \geq 0.069)$,

Table 2. Blood pressure and heart rate during dopamine infusion at three different doses in CSCI and $\mathrm{AB}$. Data are presented as mean $\pm \mathrm{SD}$ and the range.

\begin{tabular}{|c|c|c|c|c|c|}
\hline Parameter & & Pre & $1.25 \mu \mathrm{g} / \mathrm{kg} / \mathrm{min}$ & $2.5 \mu \mathrm{g} / \mathrm{kg} / \mathrm{min}$ & $5 \mu \mathrm{g} / \mathrm{kg} / \mathrm{min}$ \\
\hline \multirow[t]{4}{*}{$\mathrm{SBP}(\mathrm{mmHg})$} & CSCI* & $128 \pm 16$ & $125 \pm 14$ & $134 \pm 24$ & $147 \pm 15$ \\
\hline & & $(108-142)$ & $(112-141)$ & $(105-162)$ & $(132-168)$ \\
\hline & $\mathrm{AB}$ & $123 \pm 12$ & $120 \pm 10$ & $119 \pm 8$ & $127 \pm 13$ \\
\hline & & $(108-138)$ & $(107-130)$ & $(106-128)$ & $(115-151)$ \\
\hline \multirow[t]{4}{*}{ DBP (mmHg) } & $\mathrm{CSCI}$ & $73 \pm 10$ & $73 \pm 10$ & $74 \pm 12$ & $79 \pm 8$ \\
\hline & & $(64-87)$ & $(63-85)$ & $(60-86)$ & $(72-92)$ \\
\hline & $\mathrm{AB}$ & $72 \pm 8$ & $69 \pm 9$ & $69 \pm 6$ & $66 \pm 5$ \\
\hline & & $(64-84)$ & $(61-86)$ & $(64-82)$ & $(61-76)$ \\
\hline \multirow[t]{4}{*}{ Heart rate (bpm) } & CSCI & $64 \pm 9$ & $72 \pm 14$ & $73 \pm 11$ & $68 \pm 8$ \\
\hline & & $(57-77)$ & $(56-87)$ & $(60-86)$ & $(60-77)$ \\
\hline & $\mathrm{AB}^{*}$ & $66 \pm 7$ & $68 \pm 11$ & $71 \pm 10$ & $84 \pm 16$ \\
\hline & & $(59-76)$ & $(55-86)$ & $(62-91)$ & $(67-112)$ \\
\hline
\end{tabular}

223

Abbreviations; SBP: systolic blood pressure; DBP: diastolic blood pressure; CSCI: cervical spinal cord injury; AB: able-bodied. * Effect of time $(p<0.05)$

Blood pressure and heart rate during dopamine infusion are shown in Table 2. There was an effect of time for systolic blood pressure in CSCI only ( $p=0.038, \mathrm{AB} p=0.145)$; although pairwise comparisons only showed a trend for a difference with resting systolic blood pressure at an infusion rate of $5 \mu \mathrm{g} / \mathrm{kg} / \mathrm{min}(p=0.068)$. Three of the four participants with CSCI showed an increase in systolic blood pressure from rest to $5 \mu \mathrm{g} / \mathrm{kg} / \mathrm{min}$ of more than $20 \mathrm{mmHg}$ : 23,25 and $27 \mathrm{mmHg}$. Diastolic blood pressure did not change in either group (CSCI $p=0.272 ; \mathrm{AB} p=0.457)$, while heart rate increased in $\mathrm{AB}$ only ( $p=0.024$; $\mathrm{CSCI} p=0.348)$; with a higher heart rate during dopamine infusion at $5 \mu \mathrm{g} / \mathrm{kg} / \mathrm{min}$ compared with that at rest $(p=0.046)$. 
This pilot study investigated the acute response of lipid markers, ketone bodies and cytokines to dopamine infusion in people with CSCI. Dopamine infusion led to a sustained elevation in triglyceride and ketone body concentration in CSCI. Interleukin-6 and IL-1ra concentration, on the other hand, were not elevated after dopamine infusion in either CSCI or AB. CSCI, the elevation in the concentration of markers of lipid and ketone body metabolism (triglycerides, total cholesterol, acetoacetic acid and total ketone bodies) following dopamine administration was similar between CSCI and AB. The observed responses are in corroboration with previous studies investigating $\mathrm{AB}$ individuals $[9,20]$. After dopamine infusion for $150 \mathrm{~min}$ at rates of 3 and $6 \mu \mathrm{g} / \mathrm{kg} / \mathrm{min}$, Ensinger et al. [20] observed an acute increase in free fatty acids, while the same was found during a $5 \mathrm{~h}$ dopamine infusion protocol at $7 \mu \mathrm{g} / \mathrm{kg} / \mathrm{min}$ [9]. The similar acute response of lipids and total ketone bodies in persons with decentralised tissue below the lesion suggests that supraspinal autonomic control is not essential for lipid metabolism. This is corroborated by findings from Karlsson et al. [5,21], who found similar postprandial lipolytic rates in persons with CSCI and $\mathrm{AB}$ individuals [5] and similar fat cell characteristics in adipose tissue below and above the lesion [21]. Moreover, people with paraplegia, for whom at least part of the abdominal subcutaneous adipose tissue and in some instances the liver is decentralised whilst sympathetic input to the adrenal medulla remains intact [6], have a similar acute triglyceride response to a high-fat meal compared with $\mathrm{AB}$ persons [22]. It has also been noted, however, that sympathetic nerve activity and noradrenaline spillover following peripheral stimulation below the lesion may compensate for the lack of central sympathetic input to metabolic tissue [22]. While Karlsson [22] performed bladder percussion as a stimulus, stimulation of the sympathetic nerves may have also occurred after dopamine infusion in the people with CSCI of the present study.

Notwithstanding the potential role of peripheral stimulation and noradrenaline spill-over, the increase in circulating catecholamines is the most likely explanation for the elevated triglyceride and ketone body concentration in CSCI. Interestingly, plasma adrenaline concentration was increased in 
CSCI but not in AB. We can only speculate on the reason for this discrepancy. It may be related to a difference in adrenaline uptake between both groups. While no direct evidence is available for this supposition, a reduced clearance of catecholamines has previously been put forward as a potential reason for the exacerbated blood pressure response following noradrenaline infusion in people with CSCI and may be related to a lowered adrenergic receptor density on peripheral tissue such as the vasculature [10].

While not directly investigated in the present study, the increase in markers of lipid metabolism observed in CSCI suggests that peripheral dopaminergic and adrenergic signalling pathways remain intact following a spinal injury or undergo adjustments to compensate for the lack of intact sympathetic control. Studies investigating the vasculature and skeletal muscle tissue following SCI partially corroborate this suggestion, with a similar vasoconstrictive response to infusion of an $\alpha$ adrenergic agonist in people with SCI compared with AB persons [24] and a maintained responsiveness to $\beta$-adrenergic agonists as evidenced by skeletal muscle hypertrophy after formoterol treatment in mice with SCI [25]. Moreover, a larger increase in blood pressure following noradrenaline administration in persons with CSCI (when compared with able-bodied individuals) has been reported, potentially resulting from hypersensitivity of $\alpha$-adrenergic receptors in the vasculature [10]. The present study, however, provides no support for an exacerbated response to dopamine in persons with CSCI with regards to lipid metabolism. Systolic blood pressure, on the other hand, was elevated to a larger extent in CSCI compared with AB. While the average increase in sytolic blood pressure in CSCI did not reach the definition of autonomic dysreflexia (i.e. an increase in systolic blood pressure greater than $20 \mathrm{mmHg}$ ) [6], inspection of the individual data revealed that the increase in systolic blood pressure exceeded this cut-off in three out of the four participants with CSCI. Although no additional symptoms were observed in these individuals, this indicates that future studies should carefully consider the dopamine infusion dose. Nonetheless, future studies could investigate the effects of sympathetic nerve decentralisation on adrenergic receptor density and sensitivity in tissue important for metabolism (e.g. adipose tissue, skeletal muscle, liver and pancreas) to provide further insight into potential compensatory adjustments in peripheral tissue after CSCI. 
Ketone bodies are produced by the liver, with free fatty acids released from adipose tissue as their primary substrates. As ketone bodies can be utilised by peripheral tissue as energy source, including skeletal muscle, circulating ketone body concentrations reflect the balance between hepatic production and peripheral uptake [12]. There may therefore be several explanations for the larger increase in 3-hydroxybutyric acid in CSCI compared with AB. As persons with CSCI generally have larger stores of visceral adipose tissue [26], there may have been a higher abundance of free fatty acids to act as substrate for ketogenesis. However, although free fatty acids were not measured in the present study, concentrations of triglycerides and total cholesterol did not differ between both groups. A more likely explanation is therefore the loss of muscle mass as well as the reduced oxidative capacity of skeletal muscle in persons with CSCI [27], potentially limiting the rate of peripheral uptake of ketone bodies. Indeed, chronic endurance exercise training and a higher abundance of type 1 skeletal muscle fibres enhance the rate of ketone body clearance from the circulation after exercise [28]. Although a chronically large elevation of ketone body concentration can negatively impact on vascular and metabolic health [12], the elevated 3-hydroxybutyric acid concentration observed in CSCI is not in a range that would induce ketoacidosis [12]. In fact, a modest elevation in circulating ketone body concentration through for instance diet manipulation may induce positive effects on oxidative stress and neuronal health [12]. Future studies may investigate the effects of elevated ketone bodies concentrations on health outcomes in persons with CSCI as well as the efficacy of skeletal muscle conditioning through for example functional electrical stimulation-assisted cycling on the rate of ketone body clearance in this population.

The absence of an acute increase in IL- 6 and IL-1ra concentration may be explained by the relatively low dopamine doses used in the present study. As the dampened cytokine response to exercise in people with CSCI is strongly associated with catecholamine concentration [17], it was hypothesised that catecholamine administration alone, activating $\beta$-adrenergic receptors on skeletal muscle, would increase circulating cytokine concentrations [14]. Indeed, in resting AB individuals, infusion of adrenaline at similar concentrations as observed during endurance exercise increases IL-6 concentration [14]. However, similar to its larger impact on metabolism [9], it may be that adrenaline 
is a more potent inducer of the IL-6 response compared with dopamine. The adrenaline concentration in CSCI increased only $\sim 1.5$ fold as a result of dopamine infusion, compared with a $\sim 4$-fold increase after adrenaline infusion in the investigation by Steensberg et al. [14]. In addition, the infusion duration may have been too short to elevate IL-6 concentrations. Although Søndergaard et al. [29] found a $\sim 2$-fold increase in IL- 6 concentration after $45 \mathrm{~min}$, others observed the first increase in IL-6 concentration not until after $1.5 \mathrm{~h}$ of adrenaline infusion [14]. The low to moderate dopamine doses in this pilot study were chosen for safety reasons in this clinical population and may be an additional reason for the absence of an acute cytokine response. Furthermore, it is noteworthy that the IL-6 response to adrenaline infusion is lower when compared with a similar increase in adrenaline concentration induced by exercise [14]. Muscle contraction (and energy depletion) may therefore be needed to optimise the effects of catecholamines on IL-6 production in skeletal muscle [30]. Future studies could investigate the cytokine response to catecholamine administration combined with exercise in persons with CSCI, potentially enhancing the anti-inflammatory effects of exercise in this population [16].

Some limitations of this study need mentioning. First, as a result of difficulties with recruitment of people with CSCI for an invasive study such as the present one, the sample size is relatively small. Unfortunately, two participants with CSCI had to be excluded based on elevated baseline glucose and insulin concentrations. However, as the observed patterns in the metabolic markers were homogeneous within both groups, a lack of statistical power seems not to have influenced the findings. Although dopamine was infused at three different rates (i.e. 1.25, 2.5 and 5 $\mu \mathrm{g} / \mathrm{ml} / \mathrm{kg}$ ), blood samples were collected prior and following the complete infusion period only. This phased approach was chosen for safety reasons. As a result, however, no inferences can be made on the individual effects of the different dopamine doses. Furthermore, future studies should consider performing the experiments following an overnight fast, as the lack of nutritional control in the period prior to the $5 \mathrm{~h}$ fast before the laboratory visit may have influenced the findings [22]. Finally, the omission of additional markers of lipid metabolism, such as free fatty acids and glycerol but also fat oxidation derived from online-respirometry, limits the conclusions that can be derived from the 
present study. Future studies may include these markers and/or use more direct assessments (e.g.

343 subcutaneous microdialysis) to evaluate lipid metabolism in response to catecholamine administration 344 in persons with CSCI.

In conclusion, dopamine infusion led to a sustained elevation of triglyceride, total cholesterol and ketone body concentration in persons with CSCI, while it did not increase circulating cytokine concentration. The lack of differences between CSCI and AB in the acute response of all metabolic markers apart from 3-hydroxybutyric acid indicate that circulating catecholamines can stimulate metabolism in persons with CSCI despite the presence of autonomic dysfunction.

The dataset generated during the current study is available as a supplementary file.

Acknowledgements

The authors thank the participants for their participation in the study. Furthermore, the authors are grateful to Takamasa Hashizaki and Kohei Minami for conducting part of the biochemical analyses.

Statement of ethics

The study procedures were approved by the local ethics committee of Wakayama Medical University. We certify that all applicable institutional and governmental regulations concerning the ethical use of human volunteers were followed during the course of this research.

The authors declare no potential conflict of interest.

SPH has contributed to the study design, data collection, biochemical analyses, interpretation of the results and drafting of the manuscript.

YIK has contributed to the study design, data collection, biochemical analyses, interpretation of the results and drafting of the manuscript.

TM has contributed to the study design, data collection and drafting of the manuscript.

CAL has contributed to the interpretation of the results and drafting of the manuscript. 
$368 \mathrm{KN}$ has contributed to the study design, interpretation of the results and drafting of the manuscript.

369 VLGT has contributed to the interpretation of the results and drafting of the manuscript.

370 YN has contributed to the interpretation of the results and drafting of the manuscript.

371 YU has contributed to the study design, interpretation of the results and drafting of the manuscript.

372 MN has contributed to the interpretation of the results and drafting of the manuscript.

373 YF has contributed to the interpretation of the results and drafting of the manuscript.

374 FT has contributed to the study design, data collection, interpretation of the results and drafting of the

375 manuscript.

376 All authors have approved the final version of this manuscript and declare to be accountable for all

377 aspects of the work.

$378 \quad$ Funding

379 This project was supported by the Nachikatsuura Research Foundation (L1221; Fumihiro Tajima) and 380 the Kyoten Research Center of Sports for Persons with Impairments. 


\section{$401 \quad$ References}

402

403

404

405

406

407

408

409

410

1. Bauman WA, Spungen AM. Disorders of carbohydrate and lipid metabolism in veterans with paraplegia or quadriplegia: a model of premature aging. Metab. 1994; 43: 749-756.

2. Nash MS, Groah SL, Gater Jr DR, Dyson-Hudson TA, Lieberman JA, Myers J, Consortium for Spinal Cord Medicine. Identification and management of cardiometabolic risk after spinal cord injury: clinical practice guideline for health care providers. Top. Spinal Cord Inj. Rehabil. 2018; 24: 379-423.

3. Li X, Sun K. Regulation of lipolysis in adipose tissue and clinical significance. In: Neural regulation of metabolism Wu Q, Zheng R. (eds). (Springer Vol 1090, 1998) pp 199-205

4. Youngstrom TG, Bartness TJ. White adipose tissue sympathetic nervous system denervation increases fat pad mass and fat cell number. Am. J. Physiol. Reg. Integr. Comp. Physiol. 1998; 275: 1488-1493.

5. Karlsson AK, Attvall S, Jansson PA, Sullivan L, Lännroth P. Influence of the sympathetic nervous system on insulin sensitivity and adipose tissue metabolism: A study in spinal cord—injured subjects. Metab. Clin. Exp. 1995; 44: 52-58.

\section{Krassioukov A, Warburton DE, Teasell R, Eng JJ, Spinal Cord Injury Rehabilitation Evidence Research} Team. A systematic review of the management of autonomic dysreflexia after spinal cord injury. Arch. Phys. Med. Rehab. 2009; 90: 682-695.

7. Lorenzi M, Karam JH, Tsalikian E, Bohannon NV, Gerich JE, Forsham PH. Dopamine during $\alpha$-or $\beta$ adrenergic blockade in man: hormonal, metabolic and cardiovascular effects. J. Clin. Invest. 1979; 63: 310-317.

8. Van Loon GR, Sole MJ. Plasma dopamine: source, regulation, and significance. Metab. 1980; 29: 1119-1123.

9. Ensinger H, Weichel T, Lindner KH, Grünert A, Georgieff M. Are the effects of noradrenaline, adrenaline and dopamine infusions on VO2 and metabolism transient? Intens. Care Med. 1995; 21: 50-56.

10. Mathias CJ, Frankel HL, Christensen NJ, Spalding JM. Enhanced pressor response to noradrenaline in patients with cervical spinal cord transection. Brain J. Neurol. 1976; 99: 757-770.

11. Pernet A, Hammond VA, Blesa-Malpica G, Burrin J, Ørskov H, Alberti KGMM, Johnston DG. The metabolic effects of dopamine in man. Eur. J. Clin. Pharm. 1984; 26: 23-28.

12. Puchalska P, Crawford PA. Multi-dimensional roles of ketone bodies in fuel metabolism, signaling, and therapeutics. Cell Metabol. 2017; 25: 262-284. 

production in human keratinocytes. Neuroimmunomodulat. 2012; 19: 359-366. 14. Steensberg A, Toft AD, Schjerling P, Halkjær-Kristensen J, Pedersen BK. Plasma interleukin-6 during strenuous exercise: role of epinephrine. Am. J. Physiol. Cell Physiol. 2001; 281: 1001-1004. 15. Steensberg A, Fischer CP, Keller C, Møller K, Pedersen BK. IL-6 enhances plasma IL-1ra, IL-10, and cortisol in humans. Am. J. Physiol. Endocrinol. Metab. 2003; 285: 433-437. 16. Pedersen BK. Muscular interleukin-6 and its role as an energy sensor. Med. Sci. Sports Exerc. 2012; 44: 392-396. inflammatory response to a wheelchair half-marathon in people with a spinal cord injury-the role of autonomic function. J. Sports Sci. 2019; 37: 1717-1724.

439 18. Dill DB, Costil DL. Calculation of percentage changes in volumes of blood, plasma, and red cells in 440 dehydration. J. Appl. Physiol. 1974; 37: 247-248. 19. Nakagawa S. A farewell to Bonferroni: the problems of low statistical power and publication bias. Behav.

442 Ecol. 2004; 15: 1044-1045. dopamine infusions on oxygen consumption in volunteers. Crit. Care Med. 1993; 21: 1502-1508. 21. Karlsson AK, Elam M, Friberg P, Biering-Sörensen F, Sullivan L, Lönnroth P. Regulation of lipolysis by the sympathetic nervous system: A microdialysis study in normal and spinal cord-injured subjects. Metab. 1997; 46: 388-394. influence of visceral fat on the postprandial lipemic response in men with paraplegia. J. Am. Coll. Nutr. 2010; 29: 476-481.

23. Karlsson AK. Insulin resistance and sympathetic function in high spinal cord injury. Spinal Cord. 1999; 37: 494-500. spinal cord-injured individuals. Circulation. 2003; 108: 2361-2367. Stimulation of Mitochondrial Biogenesis Using the Food and Drug Administration-Approved $\beta 2$ Adrenoreceptor Agonist Formoterol for the Treatment of Spinal Cord Injury. J. Neurotraum. 2019; 36: 962-972. adipose tissue are greater in adults with than in those without spinal cord injury, despite matching waist circumferences. Am. J. Clin. Nutr. 2008; 87: 600-607. 

genes essential for glucose and lipid metabolism in skeletal muscle from spinal cord injured subjects. J. Appl. Physiol. 2011; 110: 1204-1210.

464 28. Evans M, Cogan KE, Egan B. Metabolism of ketone bodies during exercise and training: physiological basis 465 for exogenous supplementation. J. Physiol. 2017; 595: 2857-2871.

466 29. Søndergaard SR, Ostrowski K, Ullum H, Pedersen BK. Changes in plasma concentrations of interleukin-6 467 and interleukin-1 receptor antagonists in response to adrenaline infusion in humans. Eur. J. Appl. Physiol.

468 2000; 83: 95-98.

469 30. Steensberg A, Van Hall G, Osada T, Sacchetti M, Saltin B, Pedersen BK. Production of interleukin-6 in 470 contracting human skeletal muscles can account for the exercise-induced increase in plasma interleukin-6. J.

471 Physiol. 2000; 529: 237-242. 
494 Fig. 1 The acute changes in plasma catecholamine concentration following dopamine infusion in CSCI (grey)

495 and $\mathrm{AB}$ (white). Lines indicate individual data, while the bars represent group means. P: post. * effect of time, \# 496 difference between groups $(p<0.05)$.

497 Fig. 2 The acute changes in serum ketone body and triglyceride concentrations following dopamine infusion in $\mathrm{CSCI}$ (grey) and $\mathrm{AB}$ (white). Lines indicate individual data, while the bars represent group means.

$499 *$ effect of time $(p<0.05)$.

500 Table 1. The acute changes in cytokine concentrations and markers of glucose, lipid and ketone metabolism 501 following dopamine infusion in CSCI and AB.

502

503

504

505

506

507

508

509

510

511

512

513

514

515

516

517

518

519

520

521

522

523

524

525

526

Table 2. Blood pressure and heart rate during dopamine infusion at three different doses in CSCI and AB. Data are presented as mean $\pm \mathrm{SD}$ and the range. 
